\title{
Effect of RNA silencing suppression activity of chrysanthemum virus B p12 protein on small RNA species
}

\author{
Ramesh R. Vetukuri ${ }^{1} \cdot$ Pruthvi B. Kalyandurg ${ }^{1,2} \cdot$ Ganapathi Varma Saripella $^{1} \cdot$ Diya Sen $^{3} \cdot$ Jose Fernando Gil $^{2}$. \\ Nina I. Lukhovitskaya ${ }^{2,4} \cdot$ Laura J. Grenville-Briggs $^{3} \cdot$ Eugene I. Savenkov $^{2} \mathbb{D}$
}

Received: 27 May 2020 / Accepted: 1 September 2020 / Published online: 10 October 2020

(c) The Author(s) 2020

\begin{abstract} miRNAs, most of which were downregulated.

$\begin{array}{ll}\text { Abbreviations } \\ \text { AGO } & \text { Argonaute } \\ \text { CVB } & \text { Chrysanthemum virus B } \\ \text { CWMV } & \text { Chinese wheat mosaic virus } \\ \text { DEM } & \text { Differentially expressed miRNA } \\ \text { GFP } & \text { Green fluorescent protein } \\ \text { PMTV } & \text { Potato mop-top virus } \\ \text { miRNA } & \text { Micro RNA } \\ \text { NGS } & \text { Next-generation sequencing } \\ \text { RDR6 } & \text { RNA-directed RNA polymerase 6 } \\ \text { RISC } & \text { RNA-induced silencing complex } \\ \text { RPM } & \text { Reads per million } \\ \text { siRNA } & \text { Small interfering RNA }\end{array}$
\end{abstract}

Chrysanthemum virus B encodes a multifunctional p12 protein that acts as a transcriptional activator in the nucleus and as a suppressor of RNA silencing in the cytoplasm. Here, we investigated the impact of p12 on accumulation of major classes of small RNAs (sRNAs). The results show dramatic changes in the sRNA profiles characterised by an overall reduction in sRNA accumulation, changes in the pattern of size distribution of canonical siRNAs and in the ratio between sense and antisense strands, lower abundance of siRNAs with a U residue at the 5'-terminus, and changes in the expression of certain

Handling Editor: Jesús Navas-Castillo.

Electronic supplementary material The online version of this article (https://doi.org/10.1007/s00705-020-04832-y) contains supplementary material, which is available to authorized users.

Eugene I. Savenkov

eugene.savenkov@slu.se

1 Department of Plant Breeding, Swedish University of Agricultural Sciences, Alnarp, Sweden

2 Department of Plant Biology, Uppsala BioCenter, Swedish University of Agricultural Sciences, Linnean Center for Plant Biology, Uppsala, Sweden

3 Department of Plant Protection Biology, Swedish University of Agricultural Sciences, Alnarp, Sweden

4 Present Address: Division of Virology, Department of Pathology, University of Cambridge, Cambridge, UK

\section{sRNAs Small RNAs}

vsiRNAs Virus-derived small interfering RNAs

VSR Viral suppressor of RNA silencing

RNA silencing has evolved as a widespread innate antiviral immunity mechanism in many eukaryotic organisms. Production of virus-derived small interfering RNAs (vsiRNAs) of 21, 22 and 24 nucleotides (nt) by host Dicer enzymes is an ubiquitous feature of any virus infection in plants [1]. vsiRNA are sorted into RNA-induced silencing complexes (RISCs), containing various Argonaute (AGO) proteins, based on the nucleotide residue at the 5'end to initiate the cleavage and destruction of cognate viral RNAs [2]. The major antiviral AGO proteins, namely AGO1 and AGO2, preferentially bind siRNAs with a $U$ and $A$ residue at the 5 ' end, respectively [2-6]. Being the targets of RNA silencing machinery, plant viruses have evolved viral suppressors of RNA silencing (VSRs) that are able to dampen the host antiviral RNA silencing defence [7-9].

The cytoplasmically replicating chrysanthemum virus $\mathrm{B}$ - a member of the genus Carlavirus within the family Betaflexiviridae - encodes a multifunctional p12 protein. Previously, we showed that the nuclear-localised fraction of the protein is involved in activation of transcription of certain genes, developmentally reprogramming the host for the benefit of the virus [10]. P12 also acts as a VSR in the cytoplasm [11], yet much work remains to be done to better understand the mechanisms of RNA silencing suppression by $\mathrm{p} 12$. The first step towards this goal would be the 
characterisation of small RNA (sRNA) profiles during p12 expression, and comparison of those to appropriate negative controls as well as to sRNA profiles obtained upon expression of a well-characterised VSR, e.g., HcPro of potyviruses.

For the past decade, high-throughput sequencing (HTS) has become a widespread method to characterise sRNAs associated with viral infections or to compare small-RNA profiles in the presence and absence of VSR expression [12-14]. However, most of these studies have focused on rather late stages of virus infection, when the virus is readily detectable in the plant (1-2 weeks post-inoculation). Here, we sought to characterise sRNAs $96 \mathrm{~h}$ after expression of the VSR, thus, mimicking the early stages of the infection process.

P12 is able to suppress RNA silencing by complementing a turnip crinkle virus lacking a VSR gene (TCV-sGFP) [11]. However, until now, there has been no analysis of sRNAs in the presence of $\mathrm{p} 12$. Thus, there is a need to determine whether and which sRNAs are targeted by p12. Furthermore, comparison of sRNA profiles between a weak (p12) and strong (HcPro) VSR could provide information as to the possible mechanisms of RNA silencing suppression. The effect of p12-mediated silencing suppression on small RNA accumulation was investigated by performing HTS of small RNAs. To this end, Nicotiana benthamiana plants were grown under long day conditions (16h light/8h dark) in growth chambers with a minimum daytime temperature of $20^{\circ} \mathrm{C}$ and a night-time temperature of $18{ }^{\circ} \mathrm{C}$. Fullyexpanded leaves of 1-month-old $N$. benthamiana plants were agro-infiltrated for co-expression of $G F P$ plus empty plasmid control (GFP/EP), or GFP plus potato-virus-A-encoded HcPro (GFP/HcPro), or GFP plus p12 (GFP/p12) (Supplementary Fig. 1). For each treatment, we used three plants (three biological replicates) with two leaves being infiltrated on each plant. Tissues from these leaves were collected prior RNA extraction.

Total RNA was isolated using a mirVana miRNA Isolation Kit (Ambion) according to the manufacturer's instructions, and the RNA quality was assessed with the aid of Agilent Bioanalyzer chips. Nine sRNA libraries were constructed using total RNA isolated from infiltrated patches of $N$. benthamiana leaves 4 days post-infiltration (dpi) and a TruSeq RNA Library Preparation Kit (Illumina, Inc.). Sequencing was performed on an Illumina HiSeq2500 instrument in high-output mode, with single reads of $1 \times 50 \mathrm{bp}$ at SciLifeLab, Stockholm. Quality assessment of raw reads was performed using FastQC v 0.11.3. Adapter trimming was carried out using cutadapt $\mathrm{v}$ 1.2.1 with a minimal sequence length of 18 after adaptor removal [15]. Cleaned reads from each library were aligned to $N$. benthamiana reference sequences or $S G F P$ using Bowtie v1.2.2, allowing two mismatches per seed (-n 2), a seed length of 18 (-1 18), and no mismatch in the read alignment (-v 0$)$ and with the rest of the alignment parameters set to their default values. SAM alignment files produced by Bowtie were converted to BAM files, sorted, and indexed using SAMtools v 0.1.19 [16]. Counts of reads per sRNA length class were obtained from each library. Additionally, sense/ antisense location, position along the reference sequence, and nucleotide preference for each length class were determined with SAMtools and custom scripts written in bash and perl. Read counts for each library were normalized into reads per million (RPM) and averaged across replicates. Differential expression analysis of annotated $N$. benthamiana miRNAs [17] was carried out with Salmon and DESeq2. Briefly, miRNA sequences were indexed and quantified using Salmon v 0.9.1, and differential expression analysis was carried out using DESeq2 (Supplementary Table 1). Graphs showing counts of sRNA along reference genes were plotted in R v 3.5.1. All other graphs were plotted in Excel v 16.16.15.

Sequencing of nine sRNA libraries resulted in 12,370,110 to $35,127,860$ cleaned high-quality reads for each sample (Table 1). As shown in Table 1, the abundance of GFPderived sRNAs was as high as $16.4 \%$ in the GFP/EP library, whereas it was below $9.6 \%$ in the GFP/HcPro libraries, or even below 3.7\% in the GFP/p12 libraries (Table 1; Fig 1a).

The sRNA read length analysis demonstrated that the sizes of siRNAs from all three treatments centred at 21, 22 and 24 nt (Fig. 1b; Supplementary Fig. 2). The general size distribution of the sRNA reads was in line with previous reports $[14,18]$. However, the normalized size distribution of total sRNAs differed between the treatments, with 24-nt species being predominant (33.2\%), followed by $21-n t$ and 22-nt sRNA populations in the GFP/EP library (Fig. 1b). On the other hand, the pattern of size distribution was different in the presence of HcPro and p12, with 21-nt species being more prevalent ( $48.8 \%$ and $39.5 \%$, respectively) than $22-n t$ and 24-nt sRNA populations (Fig. 1b).

Further analyses were performed by aligning canonical sRNAs of the 21-nt, 22-nt and 24-nt size classes to the sGFP sequence (Fig. 1c). The single-nucleotide resolution maps generated for each treatment showed that the polarity of siRNA reads from different treatments displayed huge variations (Fig. 1c). The prevalence of antisense (60.8-67.1\%) over sense-strand siRNAs (32.9-39.2\%) was observed in the GFP/EP libraries (Fig. 1d and e), suggesting efficient incorporation of the antisense strand of siRNAs into RISC. Here, they mediate cleavage of GFP mRNA by means of complementarity and, at the same time, get stabilised and protected from rapid degradation, whereas the corresponding 'passenger' sense strand gets cleaved/degraded. In contrast, in the GFP/HcPro libraries, sense strands of siRNAs were more abundant (sense, $53.5-77.8 \%$ versus antisense, $22.2-46.5 \%$ ) for all canonical sRNAs of the 21-nt, $22-n t$ and 24-nt size classes (Fig. 1d and e). Thus, expression of 
Table 1 Summary of the results of NGS of small RNAs from wt Nicotiana benthamiana leaves co-expressing GFP with either EP or HcPro or p12

\begin{tabular}{|c|c|c|c|c|c|c|c|c|}
\hline Library & Replicate & Total reads ${ }^{\mathrm{a}}$ & Reads after QC & HQ reads & $\begin{array}{l}\text { Reads mapped to } \\
N . \text { benthamiana } \\
\text { genome }\end{array}$ & $\begin{array}{l}\text { Percentage of } \\
\text { reads mapped to } \\
N . \text { benthamiana } \\
\text { genome }\end{array}$ & $\begin{array}{l}\text { Reads } \\
\text { mapped to } \\
\text { GFP }\end{array}$ & $\begin{array}{l}\text { Percentage of } \\
\text { reads mapped to } \\
\text { GFP }\end{array}$ \\
\hline \multirow[t]{3}{*}{ GFP/EP } & Replicate 1 & $35,207,536$ & $22,128,179$ & $62.85 \%$ & $12,204,784$ & $55.15 \%$ & $3,627,840$ & $16.39 \%$ \\
\hline & Replicate 2 & $31,377,982$ & $17,199,648$ & $54.81 \%$ & $9,578,609$ & $55.69 \%$ & $2,818,900$ & $16.39 \%$ \\
\hline & Replicate 3 & $29,536,107$ & $16,121,007$ & $54.58 \%$ & $9,010,874$ & $55.90 \%$ & $2,644,477$ & $16.40 \%$ \\
\hline \multirow[t]{3}{*}{ GFP/HcPro } & Replicate 1 & $16,832,919$ & $12,370,110$ & $73.49 \%$ & $6,100,105$ & $49.31 \%$ & $1,046,859$ & $8.46 \%$ \\
\hline & Replicate 2 & $48,556,722$ & $35,127,860$ & $72.34 \%$ & $17,050,815$ & $48.54 \%$ & $3,385,821$ & $9.64 \%$ \\
\hline & Replicate 3 & $26,259,510$ & $17,532,394$ & $66.77 \%$ & $8,534,076$ & $48.68 \%$ & $1,651,377$ & $9.42 \%$ \\
\hline \multirow[t]{3}{*}{$\mathrm{GFP} / \mathrm{p} 12$} & Replicate 1 & $22,489,465$ & $14,900,009$ & $66.25 \%$ & $7,779,266$ & $52.21 \%$ & 555,643 & $3.72 \%$ \\
\hline & Replicate 2 & $24,050,417$ & $14,699,018$ & $61.12 \%$ & $8,042,616$ & $54.72 \%$ & 505,232 & $3.44 \%$ \\
\hline & Replicate 3 & $26,191,281$ & $16,539,121$ & $63.15 \%$ & $9,092,689$ & $54.98 \%$ & 526,344 & $3.18 \%$ \\
\hline
\end{tabular}

HcPro markedly enhances the GFP siRNA bias toward the sense strand (Fig. 1e). This can be explained by the previously reported ability of HcPro to sequester siRNA through size-specific binding and to interfere with the methylation of siRNA. Thus, the stability of siRNAs is affected [19-21]. Similar amounts of sense and antisense 22-nt and 24-nt siRNAs were found in the GFP/p12 libraries (Fig. 1e), whereas the ratio of sense to antisense of 21-nt siRNAs was similar to the EP control samples (37.9\% to 62.1\%; Fig. 1e), suggesting little (or no) effect on 21-nt species on p12 expression. Several siRNAs hot spots were detected on the GFP transcript (Fig. 1c). Most of these hot spots clustered toward the 3'-proximal region, suggesting involvement of host RNA-dependent RNA polymerase 6 (RDR6) in converting the GFP transcript into dsRNA that is then cleaved into siRNAs. In the presence of VSRs, 21-nt and 22-nt siRNAs were predominant within these hot spots, whereas 24-nt siRNA, followed by 21-nt and 22-nt siRNAs, predominated within hot spots in the GFP/EP libraries (Fig. 1c).

Selective loading of sRNAs into specific AGOs is preferentially directed by the 5 -terminal nucleotide [2]. In light of this information, we chose to more closely examine the relative abundance of sRNAs according to the nucleotide residue at their $5^{\prime}$-terminus. To assess the base preference at the $5^{\prime}$ end, we calculated the relative frequency of each of four bases, which was then normalised by the average percentage of each base along the selected positions on the reads. We found that 21- and 22-nt siRNAs size classes with $\mathrm{U}$ residue at the $5^{\prime}$ end were less abundant $(22.7-27.5 \%)$ in the GFP/HcPro and GFP/p12 libraries than in the GFP/EP control (37.5-37.9\%), whereas there was no effect on the 24-nt siRNA size class (Fig. 1f). The base $\mathrm{C}$ was the least favoured at the $5^{\prime}$ end of 21- and 22-nt siRNAs size classes for the EP control (16.8 and $18.3 \%$, respectively) and p12
(17.3 and $18.5 \%$, respectively), whereas this bias shifted to an A residue for HcPro (21.6 and $21.5 \%$, respectively; Fig. 1f).

Differentially expressed miRNAs (DEMs) were obtained using the approach described above. In total, the expression of 53 and $55 \mathrm{~N}$. benthamiana miRNAs could be measured in GFP/p12- and GFP/HcPro-infiltrated leaves, respectively (Supplementary Fig. 3, Supplementary Table 1). After filtering $(p<0.05)$, nine miRNAs were differentially expressed in the GFP/p12 dataset, and five miRNAs in GFP/HcPro dataset (Fig. 2a, bars with asterisks; Supplementary Fig. 3, Supplementary Table 1). Only one miRNA (miR479) was differentially expressed in both datasets (Fig. 2a). Thus, a total of 13 DEMs were obtained for the VSR groups (Fig. 2a). To confirm the sRNA-seq results, the abundance levels of these 13 miRNAs were tested using a quantitative stem-loop RTqPCR [22] (Fig. 2b). A regression analysis showed a very weak positive correlation (Pearson's correlation coefficient, $r=0.20$ ) between the $\log _{2}$ fold change in the HTS data and quantification of the miRNAs by RT-qPCR (Fig 2a and b; data not shown). Interestingly, according to the stem-loop RT-qPCR, most of the miRNAs analysed (9 out of 13) were downregulated in the presence of $\mathrm{p} 12$, whereas six miRNAs were upregulated and seven were downregulated upon HcPro expression (Fig. 2b).

Additionally, we chose to verify expression levels of four miRNA using a quantitative stem-loop RT-qPCR. These miRNAs included miR166 belonging to a highly conserved miRNA family and three miRNAs predominant in Solanaceae species (miR1919, miR6025 and miR6149) [17]. The analysis showed upregulation of expression of miR166 in the presence of HcPro, but not p12 (Fig. 2c). On the other hand, miR1919 and miR6149 were strongly downregulated upon expression of p12, but slightly upregulated by HcPro. 

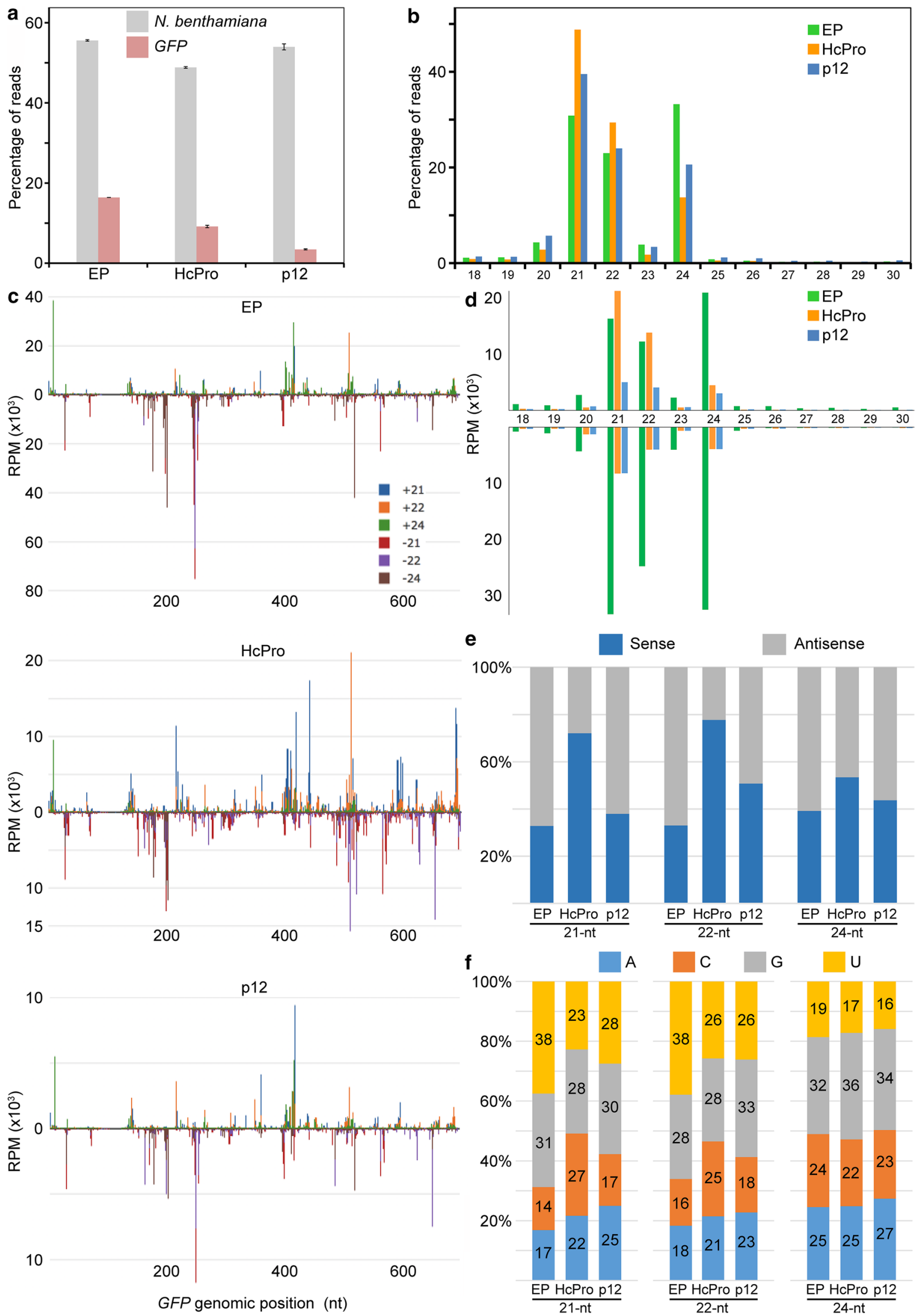
४Fig. 1 Profiles of GFP-derived small interfering RNAs (siRNAs) captured by deep sequencing from wt Nicotiana benthamiana leaves co-expressing GFP with either EP or HcPro or p12. (a) Percentage of total small RNAs (sRNAs) reads (18-30 nt) derived from the nine libraries. (b) Size distribution of 18- to 30-nt sRNAs mapped to the GFP gene. (c) Representative images of single-nucleotide resolution maps of siRNAs aligned to the GFP gene. Peaks of different colours indicate three classes (21-, 22- and 24-nt) of GFP-specific siRNAs derived from sense and antisense strands. (d) Size distribution and strand polarity of 18- to 30-nt GFP-derived siRNAs. The graphs show read count (abundance) per million mapped reads. (e) Percentage of sense and antisense $G F P$-derived siRNAs. (f) Relative frequency of the 5'-terminal nucleotide of GFP-derived siRNAs. The data represent averages of three libraries for each treatment. RPM, reads per million

MiR6025 was downregulated in the presence of both HcPro and p12 (Fig. 2c), although the relative level of reduction of miR6025 was greater with p12 expression (4.35-fold on average) than with HcPro expression (1.35-fold on average).

Using global sRNA-seq and real-time PCR analysis, we identified some differences in the accumulation of certain classes of siRNAs and miRNAs in the presence of VSRs analysed in this study. These differences included (i) an overall reduction in the number of GFP sRNA reads in the presence of VSRs relative to the EP control ( 3.2-9.6\% versus $\sim 16.4 \%$ ), (ii) a shift in the prevalence of canonical sRNA, with 24-nt siRNA being most abundant in the absence of VSR expression and 21-nt species being more abundant when VSRs were expressed, (iii) changes in the ratio between sense and antisense strands of siRNA with the prevalence of antisense over sense strand in the absence of VSR expression, whereas sense-strand siRNA were more abundant in the presence of HcPro and almost equal amounts of sense and antisense strands of the 22-nt and 24-nt size classes, but not 21-nt siRNAs, were present upon p12 expression, and (iv) lower abundance of siRNAs with a $U$ residue at the 5 ' terminus upon expression of both HcPro and p12 as compared to EP control. Supporting these observations, we recently showed that a weak VSR, the $8 \mathrm{~K}$ protein, encoded by a P1 isolate of a soil-borne potato moptop virus (PMTV) interferes with accumulation of certain classes of sRNAs [14]. Notably, the accumulation of antisense strands of 22-nt siRNAs was reduced, and the proportion of siRNAs with a 5'-terminal nucleotide residue $U$ was lower in the GFP/8K libraries than in the GFP/EP libraries [14]. The higher reduction in antisense than sense virusspecific siRNA was also observed in NbRDR6-silenced plants infected with a soil-borne Chinese wheat mosaic virus (CWMV) [12]. Interestingly, it has also been reported that the proportion of siRNAs with a $\mathrm{U}$ residue at the $5^{\prime}$ terminus in plants infected with CWMV was lower at low $\left(16^{\circ} \mathrm{C}\right)$ temperatures than in those infected at high $\left(24^{\circ} \mathrm{C}\right)$ temperatures [12]. Considering that siRNAs with a $5^{\prime}$-terminal $\mathrm{U}$ are mostly loaded into AGO1, an AGO with a major antiviral function [3-6], it is possible that a smaller proportion of siRNA are incorporated into the RISC at low temperatures or upon expression of HcPro, $8 \mathrm{~K}$, or p12 VSRs, thus dampening the antiviral response under these conditions.

Overall, our data show dramatic changes in sRNA profiles already after 96 hours of VSR expression with a clear difference between profiles generated for HcPro (a strong VSR) and p12 (a weak VSR). Further experimentation on the impact of p12 on cellular RNA silencing pathways may be a fruitful line of inquiry in the future. 
Fig. 2 Quantification of miRNAs. (a) $\log _{2}$ fold change of deferentially expressed miRNAs (DEMs) identified in the HTS data after filtering $(p<0.05)$. (b) Validation of HTS data by stemloop RT-qPCR. $\log _{2}$ relative fold expression miRNAs identified as DEMs in the HTS data. (c) $\log _{2}$ relative fold expression of three miRNAs predominant in Solanaceae species and a highly conserved miR166.

The expression levels were normalised to the expression of $N b P P 2 A$, and EP control set to value of 1 . Asterisks indicate significant statistical differences relative to EP control, ${ }^{*} p<0.05$; Student's two-tailed $t$-test


Acknowledgements This work was supported by the Swedish Research Council Formas (grant 2018-00591 to EIS; grant 2015-00430 to LGB; grant 2019-01316 to RRV), the Swedish Foundation for Strategic
Research (SSF), through a Future Research Leaders Grant (FFL5) to LGB, and Helge Ax:son Johnsons foundation. Sequencing was performed by the Science for Life Laboratory Platform in Stockholm. We 
acknowledge support from Science for Life Laboratory, the Knut and Alice Wallenberg Foundation, the National Genomics Infrastructure funded by the Swedish Research Council, and Uppsala Multidisciplinary Center for Advanced Computational Science for assistance with massively parallel sequencing (alternatively genotyping) and access to the UPPMAX computational infrastructure. We declare that we have no conflict of interests.

Funding Open access funding provided by Swedish University of Agricultural Sciences.

Open Access This article is licensed under a Creative Commons Attribution 4.0 International License, which permits use, sharing, adaptation, distribution and reproduction in any medium or format, as long as you give appropriate credit to the original author(s) and the source, provide a link to the Creative Commons licence, and indicate if changes were made. The images or other third party material in this article are included in the article's Creative Commons licence, unless indicated otherwise in a credit line to the material. If material is not included in the article's Creative Commons licence and your intended use is not permitted by statutory regulation or exceeds the permitted use, you will need to obtain permission directly from the copyright holder. To view a copy of this licence, visit http://creativecommons.org/licenses/by/4.0/.

\section{References}

1. Hamilton AJ, Baulcombe DC (1999) A species of small antisense RNA in posttranscriptional gene silencing in plants. Science (80-) 286:950-952. https://doi.org/10.1126/science.286.5441.950

2. Mi S, Cai T, Hu Y et al (2008) Sorting of small RNAs into Arabidopsis argonaute complexes is directed by the $5^{\prime}$ terminal nucleotide. Cell 133:116-127. https://doi.org/10.1016/j.cell.2008.02.034

3. Wang XB, Jovel J, Udomporn P et al (2011) The 21-nucleotide, but not 22-nucleotide, viral secondary small interfering rnas direct potent antiviral defense by two cooperative argonautes in Arabidopsis thaliana. Plant Cell 23:1625-1638. https://doi.org/10.1105/ tpc. 110.082305

4. Zhang X, Zhang X, Singh J et al (2012) Temperature-dependent survival of turnip crinkle virus-infected Arabidopsis plants relies on an RNA silencing-based defense that requires DCL2, AGO2, and HEN1. J Virol 86:6847-6854. https://doi.org/10.1128/ jvi.00497-12

5. Garcia-Ruiz H, Carbonell A, Hoyer JS et al (2015) Roles and programming of Arabidopsis ARGONAUTE proteins during turnip mosaic virus infection. PLoS Pathog 11:1-27. https://doi. org/10.1371/journal.ppat.1004755

6. Qu F, Ye X, Morris TJ (2008) Arabidopsis DRB4, AGO1, AGO7, and RDR6 participate in a DCL4-initiated antiviral RNA silencing pathway negatively regulated by DCL1. Proc Natl Acad Sci USA 105:14732-14737. https://doi.org/10.1073/pnas.0805760105

7. Sahana N, Kaur H, Jain RK et al (2014) The asparagine residue in the FRNK box of potyviral helper-component protease is critical for its small RNA binding and subcellular localization. J Gen Virol 95:1167-1177. https://doi.org/10.1099/vir.0.060269-0

8. Qu F, Ren T, Morris TJ (2003) The coat protein of turnip crinkle virus suppresses posttranscriptional gene silencing at an early initiation step. J Virol 77:511-522. https://doi.org/10.1128/ jvi.77.1.511-522.2003

9. Chen HY, Yang J, Lin C, Yuan YA (2008) Structural basis for RNA-silencing suppression by Tomato aspermy virus protein $2 \mathrm{~b}$. EMBO Rep 9:754-760. https://doi.org/10.1038/embor.2008.118

10. Lukhovitskaya NI, Solovieva AD, Boddeti SK et al (2013) An RNA virus-encoded zinc-finger protein acts as a plant transcription factor and induces a regulator of cell size and proliferation in two tobacco species. Plant Cell 25:960-973. https://doi. org/10.1105/tpc.112.106476

11. Lukhovitskaya NI, Vetukuri RR, Sama I et al (2014) A viral transcription factor exhibits antiviral RNA silencing suppression activity independent of its nuclear localization. J Gen Virol 95:2831-2837. https://doi.org/10.1099/vir.0.067884-0

12. Andika IB, Sun L, Xiang R et al (2013) Root-specific role for nicotiana benthamiana RDR6 in the inhibition of chinese wheat mosaic virus accumulation at higher temperatures. Mol Plant Microbe Interact 26:1165-1175. https://doi.org/10.1094/ MPMI-05-13-0137-R

13. Yang J, Zheng SL, Zhang HM et al (2014) Analysis of small RNAs derived from Chinese wheat mosaic virus. Arch Virol 159:3077-3082. https://doi.org/10.1007/s00705-014-2155-7

14. Kalyandurg PB, Tahmasebi A, Vetukuri RR et al (2019) Efficient RNA silencing suppression activity of Potato Mop-Top Virus 8K protein is driven by variability and positive selection. Virology 535:111-121. https://doi.org/10.1016/j.virol.2019.06.018

15. Martin M (2011) Cutadapt removes adapter sequences from highthroughput sequencing reads. EMBnet.journal 17:10. https://doi. org/10.14806/ej.17.1.200

16. Li H, Durbin R (2009) Fast and accurate short read alignment with Burrows-Wheeler transform. Bioinformatics 25:1754-1760. https ://doi.org/10.1093/bioinformatics/btp324

17. Baksa I, Nagy T, Barta E et al (2015) Identification of Nicotiana benthamiana microRNAs and their targets using high throughput sequencing and degradome analysis. BMC Genomics 16:1025. https://doi.org/10.1186/s12864-015-2209-6

18. Kutnjak D, Rupar M, Gutierrez-Aguirre I et al (2015) Deep sequencing of virus-derived small interfering RNAs and RNA from viral particles shows highly similar mutational landscapes of a plant virus population. J Virol 89:4760-4769. https://doi. org/10.1128/jvi.03685-14

19. Jamous RM, Boonrod K, Fuellgrabe MW et al (2011) The helper component-proteinase of the Zucchini yellow mosaic virus inhibits the Hua Enhancer 1 methyltransferase activity in vitro. J Gen Virol 92:2222-2226. https://doi.org/10.1099/vir.0.031534-0

20. Burgyán J, Havelda Z (2011) Viral suppressors of RNA silencing. Trends Plant Sci 16:265-272

21. Li F, Wang A (2019) RNA-targeted antiviral immunity: more than just RNA silencing. Trends Microbiol 27:792-805

22. Varkonyi-Gasic E, Hellens RP (2011) Quantitative stem-loop RTPCR for detection of microRNAs. Methods Mol Biol 744:145157. https://doi.org/10.1007/978-1-61779-123-9_10

Publisher's Note Springer Nature remains neutral with regard to jurisdictional claims in published maps and institutional affiliations. 